\title{
Strategy to Adopt and Deploy HL7 FHIR Standard for Healthcare Interoperability in Korea
}

\author{
Sungchul Bae ${ }^{1}$, Il Kon Kim ${ }^{2}$, Byoung-Kee $\mathrm{Yi}^{1,3}$ \\ ${ }^{1}$ Data Science Research Institute, Samsung Medical Center, Seoul, Korea \\ ${ }^{2}$ School of Computer Science and Engineering, Kyungpook National University, Daegu, Korea \\ ${ }^{3}$ Department of Digital Health, SAIHST, Sungkyunkwan University, Seoul, Korea
}

Recent advances in information and communication technology (ICT) have attracted attention to smart digital healthcare, which applies technology to diverse healthcare fields such as disease prevention, treatment, recovery, and rehabilitation. It is widely expected that smart digital healthcare will improve the quality of medical services and patient safety, expedite the discovery of new treatments, and further create an industrial ecosystem.

ICT-based medical technologies or services are based on the premise of collecting, exchanging, and analyzing healthrelated big data. The most important factor here is that integrated big data analysis is available only if various types of data collected from numerous sources are fused into a compatible form. For this reason, interoperability of medical information and systems is a necessary condition for healthcare big data [1].

In the healthcare field, interoperability refers to the ability to exchange medical information between medical systems or medical institutions, and to utilize the information without additional interventions. Several standards have been developed to ensure interoperability, such as HL7 (Health Level Seven International) version 2 Message, HL7 CDA (Clinical Document Architecture), and HL7 FHIR (Fast Healthcare Interoperability Resources) [2].

This is an Open Access article distributed under the terms of the Creative Commons Attribution Non-Commercial License (http://creativecommons.org/licenses/by$\mathrm{nc} / 4.0 /$ ) which permits unrestricted non-commercial use, distribution, and reproduction in any medium, provided the original work is properly cited.

(c) 2021 The Korean Society of Medical Informatics
FHIR, the next-generation health information exchange standard, has brought tremendous innovations to the health ICT field. Studies are being actively conducted to utilize FHIR for the continuity of patient medical records as well as clinical research and healthcare services. Examples include SMART on FHIR for building an Electronic Medical Records/Electronic Health Records (EMR/EHR) app ecosystem, CDS Hooks for distributed clinical decision support, CodeX as a cancer-related information exchange, FHIR Genomics as a genomic information exchange, the Da Vinci Project as a payer-based data exchange, the Gravity Project for standardization of data on social determinants of health, and the Vulcan project for connecting clinical research and healthcare $[3,4]$. In addition, other projects for clinical quality control, healthcare workflows, and healthcare big data are actively underway.

Global ICT vendors also recognize FHIR as an important part of the healthcare market and provide services using it. Cloud service giants like Amazon, Google, and Microsoft all provide FHIR interfaces for health data access to their clouds. Apple is applying FHIR to iOS so that iPhone users can receive their medical records from healthcare providers and vice versa. Epic and Cerner, the leaders in EHR systems, are also applying the FHIR to their products and are actively participating in FHIR-related activities.

The adoption of FHIR is now becoming a necessity, rather than an option. As such, policy efforts should be made and support should be provided for the national adoption and deployment of FHIR. To that end, we propose the following suggestions. First, to lay the technical foundation for the 
adoption of FHIR, it is necessary to define a list of data items to be exchanged at the national level and to create a profile of FHIR resources that correspond to them. FHIR Release 4 as a base standard is a general-purpose specification defined to meet the needs of many jurisdictions and contexts. Conversely, FHIR by itself does not satisfy the requirements of any country or a use case. To adopt it properly, the baseline FHIR resources should be profiled to reflect specific requirements, and the results should be published as a national implementation guide (IG). At present, many countries have published national IGs, including, most notably, the United States (US Core) and Australia (AuBase) as well as Canada, Switzerland, and Denmark [5-7]. To support a particular use case, an additional IG may be needed on top of the national IG.

Second, it is urgent to establish an FHIR education curriculum for developers, healthcare providers, and students to fill the vacuum of necessary expertise. A certification system and incentive policies can be considered to motivate trainees. Third, various types of FHIR-related research and development (R\&D) support are recommended for the construction of infrastructure to build a virtuous cycle of related industrial ecosystems through reviving the development of various products and services based on FHIR, and, if necessary, these initiatives should be linked to other governmentsponsored R\&D projects. Finally, it is necessary to establish a governance system to establish stable standards to help industry and consumers build trust in the standard conformance of products and services.

With advances in artificial intelligence and big data technology, recent years have shown a trend for data to become the most important resource; in particular, health data may be invaluable. Numerous research and development efforts to utilize health data are being actively conducted. Interoperability is an essential element in applying cutting-edge technologies to health data, and it is also necessary for patient- or consumer-oriented health services to ensure uniform access to health records. Globally, FHIR is regarded as a key means for ensuring interoperability of health data. In conclusion, it is imperative to keep pace with this innovation and to make the best possible efforts to adopt and deploy FHIR nationwide in Korea, as fast and adequately as possible.

\section{Notes}

HL7, CDA, and FHIR are registered trademarks of Health Level Seven International and the use of these trademarks does not constitute an endorsement by HL7.

\section{ORCID}

Sungchul Bae (https://orcid.org/0000-0002-7759-7920)

Il Kon Kim (https://orcid.org/0000-0002-2260-5241)

Byoung-Kee Yi (https://orcid.org/0000-0002-7699-9629)

\section{References}

1. Lehne M, Sass J, Essenwanger A, Schepers J, Thun S. Why digital medicine depends on interoperability. NPJ Digit Med 2019;2:79.

2. Health Lever 7 International. FHIR Release 4 [Internet]. Ann Arbor (MI): Health Lever 7 International; 2019 [cited at 2021 Jul 14]. Available from: http://hl7.org/fhir/ summary.html.

3. Boston Children's Hospital. SMART Health IT [Internet]. Boston (MA): Boston Children's Hospital; 2019 [cited at $2021 \mathrm{Jul} \mathrm{14]}$. Available from: https://smarthealthit.org.

4. HL7 International. HL7 FHIR Accelerator Program [Internet]. Ann Arbor (MI): Health Lever 7 International; 2021 [cited at 2021 Jul 14]. Available from: https://www. hl7.org/about/fhir-accelerator.

5. HL7 International. US Core Implementation Guide (STU3 Release 3.1.1) [Internet]. Ann Arbor (MI): Health Lever 7 International; 2020 [cited at 2021 Jul 14]. Available from: https://www.hl7.org/fhir/us/core.

6. HL7 Australia. Australian Base Implementation Guide (AU Base 2) [Internet]. Sydney, Australia: HL7 Australia; 2021 [cited at 2021 Jul 14]. Available from: http:// build.fhir.org/ig/hl7au/au-fhir-base.

7. Canada Health Infoway. FHIR Implementations [Internet]. Toronto, Canada: Canada Health Infoway; 2021 [cited at 2021 Jul 14]. Available from: https://infocentral. infoway-inforoute.ca/en/collaboration/wg/fhir-implementations. 